\title{
Near-peer Teaching in Histology Laboratory
}

María de los Ángeles Cambrón-Carmona, ' Carmen Natalia Tallón de Lara,' Ignacio Ruz Caracuel,' Fernando Leiva Cepas, 'Rubén Giovanetti González,' Soledad Zurita Lozano,' Juan Cámara Pérez,' Julia Casado Ruiz,' José Peña.'

About the Author: María de los Ángeles is a fifth-year medical student at University of Córdoba of a six-year program. She has been a Histology intern student for three years.

\begin{abstract}
Background: Near-peer teaching is an educational method based on being taught by one or more students who are more advanced in one specific area of the same curriculum. The aim of this study was to analyze outcomes and medical students' reactions to near-peer teaching in Histology Laboratory session. Methods: Histology Laboratory session was firstly designed as a practical session driven by academic staff, while in our new approach was driven by Histology intern students, which are upper year students in Medicine curriculum. Our near-peer teaching was evaluated using a multiple choice test when half of students had attended the session, the results of which were compared with those from traditional teaching. A reaction evaluation survey was also administered at the end of the course. Results: Multiple choice test results did not showed statistical differences between near-peer and traditional teaching strategies. Results from the reaction evaluation were mostly positive, especially with regard to feeling comfortable in the session taught by intern students and how intern students managed to transmit the information properly. Conclusion: Near-peer teaching in Histology Laboratory practical session is an effective alternative teaching method, with outcomes equivalent to traditional design, and highly valued by undergraduate medical students.
\end{abstract}

Keywords: Histology; Laboratories; Education, Medical, Undergraduate (Source: MeSH-NLM).

\section{Introduction}

Histology is one of the preclinical subjects of medical studies. It is usually taught in the first semesters, although in some problem-based learning curricula its teaching is spread across all semesters. ${ }^{1}$ The main objective of the Histology subject is to provide future physicians with the knowledge about organs' morphology at the microscopic level and its correlation with the organs' functions. Learning Histology can be accomplished by practical training using light microscope, virtual microscope or a combination of both, depending on the curriculum. ${ }^{2}$ Independent of the instrument used for visualization, the primary materials needed are histological slides. Therefore, it is important to provide some basic knowledge about how slides are prepared from specimens. For that reason, a practical session is taught inside the Medical Histology I course at the School of Medicine and Nursing, University of Córdoba (Spain).

Near-peer teaching can be defined as an educational arrangement in which one student who is more advanced in the same curriculum teaches one or more fellow students. ${ }^{3}$ It is widely regarded as an effective teaching modality, and many medical schools have implemented student-as-teacher programs that provide near-peer teaching opportunities to senior medical students. ${ }^{4}$ Moreover, there is evidence that near-peer teaching can be effective in hands-on small group practices. ${ }^{5-7}$

At our University, Histology Laboratory session was conceived as a hands-on session to better show the applicability of what is taught. It is known since the classical study by Secondy-Va- cuum Oil Co. Studies ${ }^{8}$ that students retain $30 \%$ of what they visualize versus $90 \%$ of what they explore and perform. Small groups were also more convenient for this purpose. ${ }^{9}$ Moreover, during the session, "clinical situations" related to the contents were introduced (e.g. intraoperative biopsy when processing tissues for frozen sectioning) to reinforce vertical integration. ${ }^{10}$ This teaching strategy has been demonstrated to be successful. ${ }^{11}$

Positive interactions were observed between medical students and intern students at our university, which in turn inspired the introduction of a near-peer teaching strategy in the curriculum. Our objective in the present study was to perform an outcome evaluation and a reaction evaluation in order to develop a new plan based on evidence for future use. This double evaluation consists of an evaluation of learning comparing the near-peer teaching with previous results from traditional teaching sessions and a student perception evaluation to explore students' satisfaction with the new strategy.

\section{Methods \\ Near-peer Histology Laboratory Session}

Traditional Histology Laboratory session consisted of a twohour practical session in the Histology laboratory, where students in groups of 5-6 people performed all the steps for solid tissue processing and learned how to do a cytology test of oral mucosa. Prior to this hands-on session, students were encouraged to read a 19-page document that explained different types of tissue processing depending on its source. Each session was conducted by a faculty staff with more than 20 years

${ }^{1}$ Department of Morphological Sciences, Section of Histology, Faculty of Medicine and Nursing, University of Córdoba, Córdoba, Spain. 
of teaching experience or a laboratory technician with the aid of an intern student.

In the new strategy, intern students (who are near-peers) acted not only as single instructors for the practical session, but also helped to redesign it. Changes included increasing session duration to three hours and the creation of a Prezi $®$ presentation that contained the same information previously given as a document. An attitudinal evaluation made by intern students was also included. These changes wanted to improve some lacks that were noticed, such as short time for slow explanation and poor interest in reading the aforementioned document."

\section{Study Design}

Participants were medical students who enrolled for Medical Histology I course in the 2013 Spring Semester (traditional teaching strategy, $\mathrm{n}=125$ ) and those in the 2015 Spring Semester (near-peer teaching strategy, $n=127$ ). Evaluation of learning was performed during a compulsory Histology Laboratory session for the respective cohorts when half of the cohorts had attended the session. Reaction evaluation was performed after another compulsory session for the 2015 Spring Semester cohort. While participation in the classes was compulsory, participation in the evaluations was voluntary. Intern students $(n=8)$ were students from more senior classes who voluntarily collaborated in teaching and research with faculty staff at Histology area. Six intern students were medical students (third year: $n=1$; fourth year: $n=3$; fifth year: $n=1$, sixth year: $n=1$ ). The other two intern students were a freshly graduated medical student and a third-year nursing student who was previously trained for Pathology technician.

Evaluation of learning was conducted using a multiple choice test. The test contained 10 items organized into two topics: ability of the students to select the proper methodology in a given clinical situation (Items 1-5) and knowledge of the principles of laboratory techniques (Items 6-10). Students were not aware that they were going to be evaluated, and the test was anonymous. Results were collected exclusively for this study. As evaluations were performed when half of the students had attended the Histology Laboratory session in both cohorts, there were four groups for comparison: (1) students who had attended the 2015 Spring Semester session before taking the test under the near-peer strategy (ANP), (2) students who attended the 2013 Spring Semester session before taking the test under the traditional strategy (AT), (3) students who had not attended the 2015 Spring Semester session at the time the test was administered for the near-peer strategy (NANP), and (4) students who had not attended the 2013 Spring Semester session at the time the test was administered for the traditional strategy (NAT). Participation of students in the sessions was based on the alphabetical order of students' surname; no academic criteria were applied.

Reaction evaluation for near-peer teaching was performed using a survey questionnaire about educational environment during the Histology Laboratory session. The survey consisted of eight items which were evaluated using a modified-Likert scale from 0-5 (where 0 indicated "Completely disagree" and 5 indicated "Completely agree"). Students answered questions voluntarily, knowing that the information would be used to evaluate the teaching method.

\section{Statistical Analysis}

Results from the evaluation of learning were expressed as mean \pm standard deviation. A Kruskal-Wallis one-way analysis of variance on ranks followed by Dunn's method was performed to examine the differences among groups. Statistical analysis was carried out using the Sigma Stat 3.1 software package (Systat Software Inc), and the level of statistical significance was set at $p<0.05$. A descriptive analysis was performed for reaction evaluation results.

\section{Results}

For evaluation of learning, participants were 125 students who were recruited from the 125 students enrolled for Medical Histology I course in the 2013 Spring Semester (100\% participation rate) and 100 students from the 127 enrolled in the 2015 Spring Semester ( $79 \%$ participation rate). Participants were distributed as follows: ANP, $n=60 ; A T, n=63$; NANP, $n=40$; and NAT, $n=62$ (Figure 1). Percentage of students recruited in 2015 Spring Semester was lower due to low participation rate in the NANP group. Participation rate for the reaction evaluation survey was high, with 121 out of the 127 students enrolled in the 2015 session completing the survey (95\% participation rate).

Figure 1. Study Design and Flow of Participants

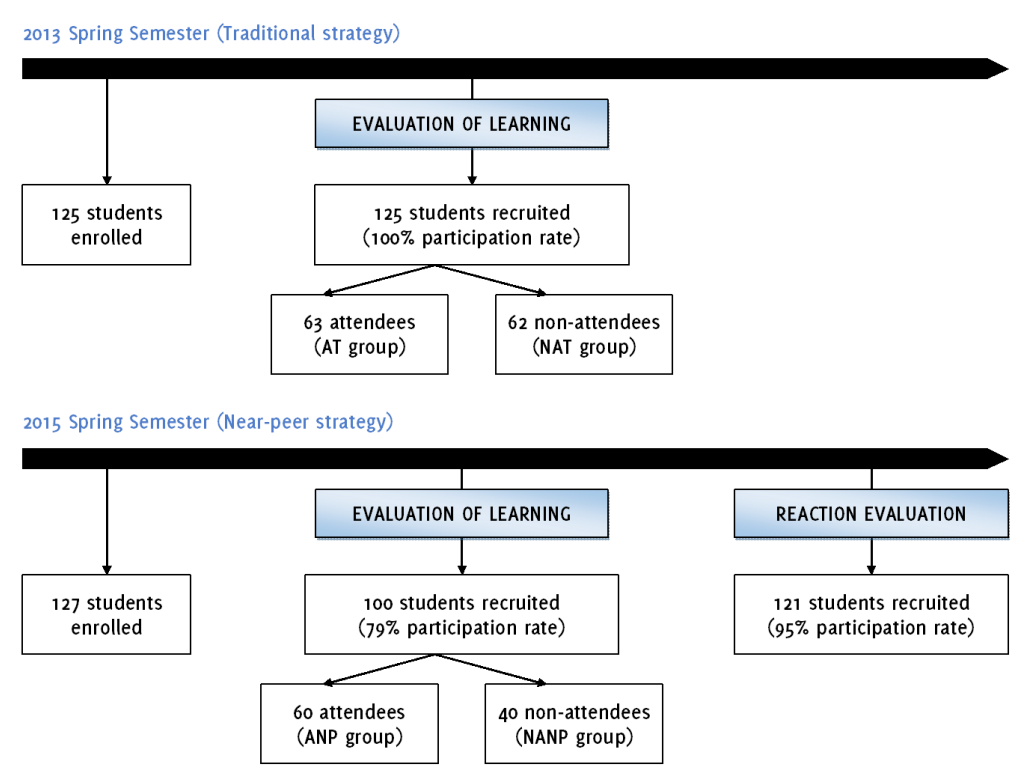

AT=attendees, traditional strategy; NAT=non-attendees, traditional strategy; ANP=attendees, near-peer strategy; NANP=non-attendees, near-peer strategy.

Multiple choice test results showed that students who have attended the Histology Laboratory sessions scored significantly higher in the clinical situation items compared with their mates who had not attended the sessions (Table 1). On the other hand, scores regarding knowledge of the principles of laboratory only showed significant differences between attendees and non-attendees in the near-peer strategy group. No differences were found between attending groups, AT and ANP, for both groups of evaluation items.

Overall, students' responses to reaction evaluation survey were 
Table 1. Evaluation of Learning at Histology Laboratory Session

\begin{tabular}{|c|c|c|}
\hline & Traditional Strategy & Near-peer Strategy \\
\hline \multicolumn{3}{|l|}{ Clinical situation items } \\
\hline Practical session attendees & $2.38 \pm 1.02(n=63)$ & $2.75 \pm 0.79(n=60)$ \\
\hline Practical session non-attendees & $1.31 \pm 0.92(n=62)^{a}$ & $0.93 \pm 0.73(n=40)^{b}$ \\
\hline \multicolumn{3}{|c|}{ Principles of laboratory techniques items } \\
\hline Practical session attendees & $4.17 \pm 0.79(n=63)$ & $3.85 \pm 0.97(n=60)$ \\
\hline Practical session non-attendees & $3.69 \pm 1.00(n=62)$ & $2.85 \pm 1.10(\mathrm{n}=40)^{\mathrm{b}, \mathrm{c}}$ \\
\hline \multicolumn{3}{|c|}{$\begin{array}{l}\text { Data are presented as mean } \pm \text { standard deviation. } \\
\text { Statistical significance between attendees and non-attendees for traditional strategy }(p<0.05) \text {. } \\
\text { Statistical significance between attendees and non-attendees for near-peer strategy }(p<0.05) \text {. } \\
\text { Statistical significance between non-attendees for traditional strategy and non-attendees for near-pe } \\
\text { strategy }(p<0.05) \text {. }\end{array}$} \\
\hline
\end{tabular}

positive (Figure 2). The best valued items were with regard to whether students felt more comfortable and relaxed in the session taught by intern students ( $89 \%$ rated 4 or 5 ) and whether information had been firmly transmitted by intern students ( $93 \%$ rated 4 or 5 ). In contrast, the least valued point was with regard to whether the students preferred the session to be delivered by intern students instead of teaching staff, with only $53 \%$ of the students answering "Completely agree" or "Strongly agree", although most of the students rated it with 3 or more out of $5(93 \%)$. Attendants also valued mostly with 4 or 5 their learning during the practical session $(96 \%)$, their confidence to ask questions $(82 \%)$, and intern students' ability to make the contents more comprehensible ( $82 \%)$. Other items not valued as high were students' confidence to take part in practical activities ( $66 \%$ rated scored 4 or 5 ) and their agreement in being evaluated by intern students ( $67 \%$ rated 4 or 5$)$.

\section{Discussion}

This study aimed to assess near-peer teaching in a laboratory practical session of Medical Histology subject and showed that learning results are equivalent to those of traditional teaching and that students valued highly session delivered by intern students. Outcome results based on multiple-choice test showed that the level of quantitative knowledge reached by students in both academic years was the same, despite the differences found in the initial results. Although we did not demonstrate that near-peer teaching is better than the traditional strategy, we have proven that teaching by intern students could be used as an alternative to teaching by academic staff in this kind of activities. It should be noted that there were some missing students in the NANP group. Because they were absent in a compulsory lesson, they would probably be less motivated students, so NANP results would more probably be overestimated than underestimated. Losses in participation could also be explained by the fact that while eligible students answered voluntarily the test, some might decline to participate.

Our results are in agreement with others comparing academic performance between near-peer teachers and staff driven small groups sessions., ${ }^{6}$, For that reason, it has been suggested that near-peer teachers could alleviate teaching pressure of faculty staff and, more interestingly, they could drive medical training programs in severely resource-constrained settings. ${ }^{13}$

Some other reasons that were considered favorably in introducing this near-peer strategy has already been highlighted; these are that near-peer tutors teach at the cognitive level of the students and develop leadership and teaching skills while the students is being taught and that learning could take place in a safe and comfortable environment. ${ }^{7}$ Although the main objective of our study was not to assess the classroom environment, ${ }^{14}$ we have showed that students valued highly and very highly the environment created by intern students, feeling comfortable, relaxed and more prone to ask questions as well as taking part in practical activities. It should be noted that social interactions that teacher-learners are exposed to are linked to their

Figure 2. Students' Reaction Evaluation to Histology Laboratory Session

1. Have you learned in the Histology laboratory session?

2. Having been taught this session by intern students, you felt more comfortable and relaxed.

3. Having been taught this session by intern students, you felt more confident to ask questions.

4. Having been taught this session by intern students, you were more confident to take part in the practical activities.

5. You consider that the language and the explanations employed by intern students made the contents more comprehensible.

6. You think the information was firmly transmitted by intern students.

7. You agree to be evaluated by intern students.

8. You prefer that the Histology laboratory session is given by intern students instead of teachers of the subject.

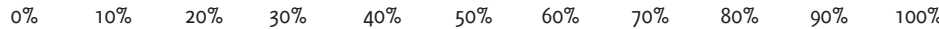

5: Completely agree $\quad$ 4: Strongly agree $\quad$ :3: Agree $\quad$ 2: Disagree $\quad$ 1: Strongly disagree $\quad$ 0: Completely disagree 
motivation to learn and that teachers' attitudes can modify students' behavior and extrinsic motivation. ${ }^{15}$ In this context, students' lower inhibition to make mistakes could be explained by the comfort and mitigation of pressure that involves receiving practices by seniors students, ${ }^{16}$ who behave similar to students.

It was interesting that the best valued item was that information was firmly transmitted by the intern student, in contrast with what has been described in relation with the lack of confidence of near peer teachers in their role of educator. ${ }^{17}$ Given this discrepancy, it would be of great interest to assess near-peer teachers' perceptions through a questionnaire as there could be a contrast between what intern students feel and learners' point of view.

Positive students' opinion to the item regarding intern students ability to make contents more comprehensible could be explained by the cognitive congruence theory, which "argues that the near-peer teachers have a better understanding of the fund of knowledge, to include shortcomings of knowledge, of their more junior student colleagues, enabling them to better clarify problems at an appropriate level", ${ }^{17}$ as has already been proven in some near-peer teaching experiences. ${ }^{18}$

Nevertheless, the item related to students' satisfaction about laboratory session given by intern students was marked worse than expected, while in the remaining items intern students were evaluated in a positive way. For that reason, a factor not included in our analysis could have caused students to have this decision. One possibility could be the fact that intern students did not fully have the role of teacher. However, the aforementioned factor could be positive in some aspects, as students perceive senior students not as faculty experts who have the authority to punish their behavior but as peers who can offer support and be even considered as friends. ${ }^{9}$ This perception could also offer some explanations for the relatively less positive results for the item on agreement in being evaluated by intern students.

Although our students did not uniformly choose near-peer teachers instead of staff teachers, our positive results can be put in relation with those reported in the literature,, 917 in which students prefer near-peer teaching. Caution for generalization should be made because most of results are taken from specific designs. ${ }^{18}$

Although our study did not analyze intern students' perspective, peer teaching could also provide some benefits to the student teachers, who could consolidate their knowledge as they prepare content, gain teaching experience, receive recognition for their teaching experience and often receive formal teacher training. ${ }^{19}$ They also may become more effective communicators, training a key ability for physician-patient interaction. ${ }^{15}$

We recognize several limitations in our study. Increasing the length of the practical session and the use of Prezi® presentation could have influenced results and complicated the comparisons. Prezi $\circledast$ encourages learners to identify patterns, comparisons, relationships, and differences between information. It can be used to emphasize the hierarchical nature of information and the connections within topics, exploring ideas and forming connections between them to form a three-dimensional mindmap. ${ }^{20,21}$ Nevertheless, in our opinion these changes should be view as part of intern students' involvement in session redesign.

Another limitation of our study is intern student heterogeneity. Intern students had different experience in laboratory, since some had recently joined while others had spent several years assisting in the laboratory. They have different experiences in terms of previous laboratory session teaching as well as level of knowledge both as intern and medical students (intern students' academic years spread from third year to recent graduate). Those different levels of experience can result in differences among students' groups.

Although our results were obtained from a single university and cannot be directly generalized, we recommend to put into practice this kind of practical session, given the constant positive results in the literature concerning near-peer teaching in small groups. ${ }^{6,7,12,19}$ Our results are especially relevant for those who want to use near-peer teaching in Histology and related areas. Our model employing intern students instead of academic staff is very useful in settings with shortage of teachers. It can be applied in universities where teachers are involved in other activities such as research, or where there is a structural deficit, like in many developing universities. Although most of the studies support near-peer teaching in small groups, some have proven advantages of this strategy in bigger groups. ${ }^{19}$

In conclusion, this study demonstrates that near-peer teaching by intern students is an alternative effective teaching in Histology Laboratory session. Outcome evaluation did not show differences with staff teaching and students' reaction evaluation results were positive, especially with regard to students' comfortable and confident feelings during sessions delivered by near-peer teachers. However, some factors which might influence students' attitudes towards choosing near-peer teachers or academic staff should be further investigated. Future educational development and research should evaluate near-peer teaching in other practical session settings and characterize the attitudes of students, near-peers and staff teachers towards the introduction of such new strategy.

\section{References}

1. Nouns Z, Schauber S, Witt C, Kingreen H, Schüttpelz-Brauns K. Development of knowledge in basic sciences: a comparison of two medical curricula. Med Educ. 2012 Dec;46(12):1206-14.

2. Bloodgood RA, Ogilvie RW. Trends in histology laboratory teaching in United States medical schools. Anat Rec B New Anat. 2006 Sep;289(5):169-75.

3. Ten Cate 0 , Durning S. Dimensions and psychology of peer teaching in medical education. Med Teach. 2007 Sep;29(6):546-52.

4. Andrew Jay E, Starkman SJ, Pawlina W, Lachman N. Developing medical students as teachers: an anatomy-based student-as-teacher program with emphasis on core teaching competencies. Anat Sci Educ. 2013 NovDec;6(6):385-92.

5. Lawry GV 2nd, Schuldt SS, Kreiter CD, Densen P, Albanese MA. Teaching a screening musculoskeletal examination: a randomized, controlled trial of different instructional methods. Acad Med. 1999 Feb;74(2):199-201.

6. Tolsgaard MG, Gustafsson A, Rasmussen MB, Høiby P, Müller CG, Ringsted C. Student teachers can be as good as associate professors in teaching clinical skills. Med Teach. 2007 Sep;29(6):553-7. 
7. Blank WA, Blankenfeld H, Vogelmann R, Linde K, Schneider A. Can nearpeer medical students effectively teach a new curriculum in physical examination? BMC Med Educ. 2013 Dec;13:165.

8. De Juan Herrero J. [Introduction to university education: educational resources for teacher training]. Madrid: Dykinson S.L.; 1996. Spanish

9. Meller SM, Chen M, Chen R, Haeseler FD. Near-peer teaching in a required third-year clerkship. Yale J Biol Med. 2013 Dec;86(4):583-9.

10. Vidic B, Weitlauf HM. Horizontal and vertical integration of academic disciplines in the medical school curriculum. Clin Anat. 2002 May;15(3):233-5. 11. Peña Amaro J, Jimena Medina I, Leiva Cepas F, Ruz Caracuel I. [Strategy and learning activities in the practical teaching of medical histology in the UCO]. XIII Jornadas de Redes de Investigación en Docencia Universitaria. 2015;2089-102. Spanish

12. Burke J, Fayaz S, Graham K, Matthew R, Field M. Peer-assisted learning in the acquisition of clinical skills: a supplementary approach to musculoskeletal system training. Med Teach. 2007 Sep;29(6):577-82.

13. Ten Cate 0 , Durning $S$. Peer teaching in medical education: twelve reasons to move from theory to practice. Med Teach. 2007 Sep;29(6):591-9.

14. Fraser BJ. Classroom environment instruments: development, validity and applications. Learn Environ Res. 1998 Jan;1(1):7-33.

15. Dandavino M, Snell L, Wiseman J. Why medical students should learn how to teach. Med Teach. 2007 Sep;29(6):558-65.

16. Topping KJ. Trends in peer learning. Educ Psych. 2005 Dec;25(6):631-45.

17. Bulte C, Betts A, Garner K, Durning S. Student teaching: views of student near-peer teachers and learners. Med Teach. 2007 Sep;29(6):583-90.

18. Lockspeiser TM, O'Sullivan P, Teherani A, Muller J. Understanding the experience of being taught by peers: the value of social and cognitive congruence. Adv Health Sci Educ Theory Pract. 2008 Aug;13(3):361-72.

19. Rashid MS, Sobowale 0, Gore D. A near-peer teaching program designed, developed and delivered exclusively by recent medical graduates for final year medical students sitting the final objective structured clinical examination (OSCE). BMC Med Educ. 2011 Mar 17;11:11.

20. Strasser $N$. Using Prezi in higher education. J Coll Teach Learn. 2014;11(2):95-8.

21. Virtanen P, Myllärniemi J, Wallander H. Diversifying higher education: facilitating different ways of learning. Campus-Wide Information Systems. 2013;30(3):201-11.

\section{Acknowledgments}

The authors are grateful to Professor Ignacio Jimena Medina for his unconditional support and Mr. Antonio Agüera for all technical support and assistance.

Conflict of Interest Statement at Funding

The Authors have no funding, financial relationships or conflicts of interest to disclose.

\section{Author Contributions}

Conceptualization: MACC, CNTL, IRC, FLC, JP. Data collection: MACC, CNTL, IRC, FLC, RCG, SZL, JCP, JCR. Data analysis and interpretation: MACC, CNTL, IRC, FLC, RGG, JP. Writing: MACC, CNTL, IRC. Critical revision of the manuscript: FLC, RGC, SZL, JCP, JCR, JCP. Approval of the final version: All authors. Administrative/technical advice: FLC, RGG.

\section{Cite as:}

Cambrón-Carmona MA, Tallón de Lara CN, Ruz Caracuel I, Leiva Cepas F, Giovanetti Conzález R, Zurita Lozano S, et al. Near-peer teaching in Histology laboratory. Int J Med Students. 2016 Jan-Apr;4(1):14-8. 